\title{
Blow-up Versus Global Well-posedness for the focusing INLS with inverse-square potential
}

\author{
Mingming Deng ${ }^{1}$, Jing $\mathrm{Lu}^{2}$, and Fanfei Meng ${ }^{3}$ \\ ${ }^{1}$ Graduate School of China Academy of Engineering Physics \\ ${ }^{2}$ China Agricultural University \\ ${ }^{3}$ Institute of Applied Physics and Computational Mathematics
}

June 7, 2021

\begin{abstract}
We study the focusing inhomogeneous nonlinear Schr $\backslash$ "odinger equation with inverse-square potential $\$ \$$ i $\backslash$ partial_tu $+\backslash$ Delta $\mathrm{u}-\backslash \operatorname{frac}\{\mathrm{a}\}\left\{|\mathrm{x}|^{\wedge} 2\right\} \mathrm{u}+|\mathrm{x}|^{\wedge}\{-\mathrm{b}\}|\mathrm{u}|^{\wedge}\{2\} \mathrm{u}=0, \$ \$$ where $\$ \mathrm{a}>-\backslash$ frac $14 \$$ and $\$ 0$
\end{abstract}

\section{Hosted file}

Blow-up Versus Global Well-posedness for the focusing INLS with inverse-square potential.pdf available at https://authorea.com/users/418321/articles/525096-blow-up-versus-global-wellposedness-for-the-focusing-inls-with-inverse-square-potential 